\title{
Genome-wide survey and phylogeny of S-Ribosylhomocysteinase (LuxS) enzyme in bacterial genomes
}

\author{
Rajas M. Rao ${ }^{1,2}$, Shaik Naseer Pasha ${ }^{1}$ and Ramanathan Sowdhamini ${ }^{*^{*}}$
}

\begin{abstract}
Background: The study of survival and communication of pathogenic bacteria is important to combat diseases caused by such micro-organisms. Bacterial cells communicate with each other using a density-dependent cell-cell communication process called Quorum Sensing (QS). LuxS protein is an important member of interspecies quorumsensing system, involved in the biosynthesis of Autoinducer-2 (AI-2), and has been identified as a drug target. Despite the above mentioned significance, their evolution has not been fully studied, particularly from a structural perspective.

Results: Search for LuxS in the non-redundant database of protein sequences yielded 3106 sequences. Phylogenetic analysis of these sequences revealed grouping of sequences into five distinct clusters belonging to different phyla and according to their habitat. A majority of the neighbouring genes of LuxS have been found to be hypothetical proteins. However, gene synteny analyses in different bacterial genomes reveal the presence of few interesting gene neighbours. Moreover, LuxS gene was found to be a component of an operon in only six out of 36 genomes. Analysis of conserved motifs in representative LuxS sequences of different clusters revealed the presence of conserved motifs common to sequences of all the clusters as well as motifs unique to each cluster. Homology modelling of LuxS protein sequences of each cluster revealed few structural features unique to protein of each cluster. Analyses of surface electrostatic potentials of the homology models of each cluster showed the interactions that are common to all the clusters, as well as cluster-specific potentials and therefore interacting partners, which may be unique to each cluster.

Conclusions: LuxS protein evolved early during the course of bacterial evolution, but has diverged into five subtypes. Analysis of sequence motifs and homology models of representative members reveal cluster-specific structural properties of LuXS. Further, it is also shown that LuxS protein may be involved in various protein-protein or proteinRNA interactions, which may regulate the activity of LuxS proteins in bacteria.
\end{abstract}

Keywords: Genome wide survey, Phylogeny, Homology modelling, LuxS protein

\section{Background}

Communication between individuals is a critical factor that decides the survival of a population. It is a pivotal factor for the survival of pathogenic and nonpathogenic bacteria alike, the diseases caused by the former being a major health concern, particularly in developing countries. The above objective is achieved in bacteria by means of a cell-to-cell communication process, involving chemical signals called Quorum-

\footnotetext{
* Correspondence: mini@ncbs.res.in

${ }^{1}$ National Centre for Biological Sciences, Tata Institute of Fundamental

Research, GKVK campus, Bellary Road, Bangalore 560065, India

Full list of author information is available at the end of the article
}

Sensing (QS). The process of QS involves the bacterial cell producing chemical signals known as Autoinducers (AIs) [1], which are secreted into the extracellular space.

Till date, three types of autoinducers have been characterised in bacteria: AI-1, AI-2 and AI-3. AI-2 is involved in inter-species cell-cell communication [2], and it was found to be a furanosyl-borate diester, making it the only boron-containing biomolecule characterised till date [3]. Even though AI-2 is observed to contain this element, its presence is highly dependent on the growth conditions of bacteria. 
Biosynthesis of AI-2 involves a three-step reaction, which is part of a methionine catabolism cycle, known as Activated Methyl Cycle (AMC). First step involves removal of methyl group from S-Adenosyl Methionine (SAM), which is catalysed by SAMdependent methyltransferases. Resulting product, SAdenosyl Homocysteine (SAH), is converted to SRibosyl Homocysteine (SRH) by the enzyme SAH Nucleosidase [4]. SRH, in turn, is hydrolysed to 4,5dihydroxy-2,3-pentanedione (4,5-DPD) by the enzyme S-Ribosylhomocysteinase, also referred to as LuxS protein [5]. 4,5-DPD further undergoes hydrolysis autocatalytically to form AI-2 [2].

An important enzyme involved in AI-2 biosynthesis is S-Ribosylhomocysteinase, also referred to as LuxS protein. This enzyme belongs to LuxS/MPP-like metallohydrolase superfamily according to SCOP system of protein classification. A remarkable feature of this protein is that it is one of the few enzymes capable of cleaving thioether bonds without using a redox cofactor [6]. Moreover, studies on LuxS gene in E. coli, V. cholerae and S. typhi have shown that the gene is highly conserved in different species, but do not share any homology with other gene [7].

Numerous structural studies have been performed on LuxS protein. The first attempts to obtain a crystal structure of LuxS protein $[8,9]$ showed the LuxS protein was a homodimer, retaining eight stranded $\beta$-barrel surrounded by six alpha-helices. The active site consists of a zinc-ion, coordinated by residues His 54 , His 58 and Cys126, which are all highly conserved. It was also observed that access to the active site seems to be restricted and is triggered by conformational changes in the protein, involving residues $125-131$ and the residues around $\mathrm{N}$-terminus.

Previous studies on evolution of LuxS protein showed that LuxS had evolved early during the divergence of major prokaryotic phyla, based on its broad consensus with single subunit ribosomal RNA tree of bacteria. However, it was inferred that there were instances of horizontal and lateral gene transfer [10]. Another genome-wide survey on LuxS genes in various bacterial genomes have shown that LuxS gene is widespread across the bacterial domain, and AI-2 mediated signalling may indeed be interspecies universal mode of cell-cell communication system [11]. In contrary to these reports, in a study that examined the AI-2 binding receptors, the authors suggested that AI2 mediated QS is restricted to certain members of Vibrionales, and to some members of pathogenic gut bacteria, and that the role of LuxS protein is limited to AMC [12].

However, with the advent of large-scale bacterial genome sequencing projects, and increasing recognition of the role of LuxS in growth and virulence of various bacterial pathogens, a broader perspective is required on the evolution of QS systems augmented with structural data. Thus, this study aims to examine the evolution of LuxS protein on a phylogenetic, as well as structural perspective.

\section{Results and discussion}

\section{Genome-wide survey of LuxS protein sequences}

LuxS homologues were searched using Hidden Markov Models [13]. The number of hits retrieved from the sequence search is 3106 and the phyletic distribution is shown in Additional file 1. High abundance of LuxS protein sequences in phyla such as Actinobacteria, Firmicutes, Gamma-Proteobacteria and Bacteroidetes could be observed. However, no LuxS homologues could be identified in many phyla, such as Chloroflexi, Aquificae, Thermotogales, Cyanobacteria etc. (Additional file 2). LuxS homologues could not be identified in certain pathogenic bacteria, such as Mycobacterium tuberculosis, Chlamydia trachomatis, Mycoplasma pneumoniae, and in Archaea, using the current search protocol. However, genome-wide survey of Pfs-protein, another protein involved in AMC that catalysed conversion of S-Adenosyl Homocysteine to S-Ribosyl Homocysteine, conducted with similar parameters using phmmer search program [13], yielded about 8000 positive hits (data not shown). Discrepancy in the number of hits, despite both of the proteins being a part of the same metabolic pathway, suggests the possibility of other enzymes playing a similar role as LuxS in organisms where no homologues could be observed.

\section{Phylogeny of LuxS protein}

We performed phylogenetic analysis of 3106 LuxS protein sequences identified from different bacterial genomes. The phylogenetic tree showed grouping of sequences into five distinct clusters, which has not been reported in previous studies to the best of our knowledge (Fig. 1a and b). The absence of phylumspecific clusters and co-clustering of LuxS sequences of different bacterial phyla confirms previous reports of high degree of conservation of LuxS sequences among different bacterial species [10]. Taxonomic distribution of LuxS protein shows grouping of Proteobacterial sequences in the fifth cluster. The Firmicute sequences are spread throughout the remaining five clusters, while Actinobacterial sequences are distributed in second and third clusters. The sequences of Bacteroidetes, Spirochaetes, Fusobacteria are present in Cluster-1, while the sequences of phylum Dienococcus-Thermales is present in the Cluster-4. (Additional files 3 and 4). There are a few cases where LuxS protein may have evolved by 


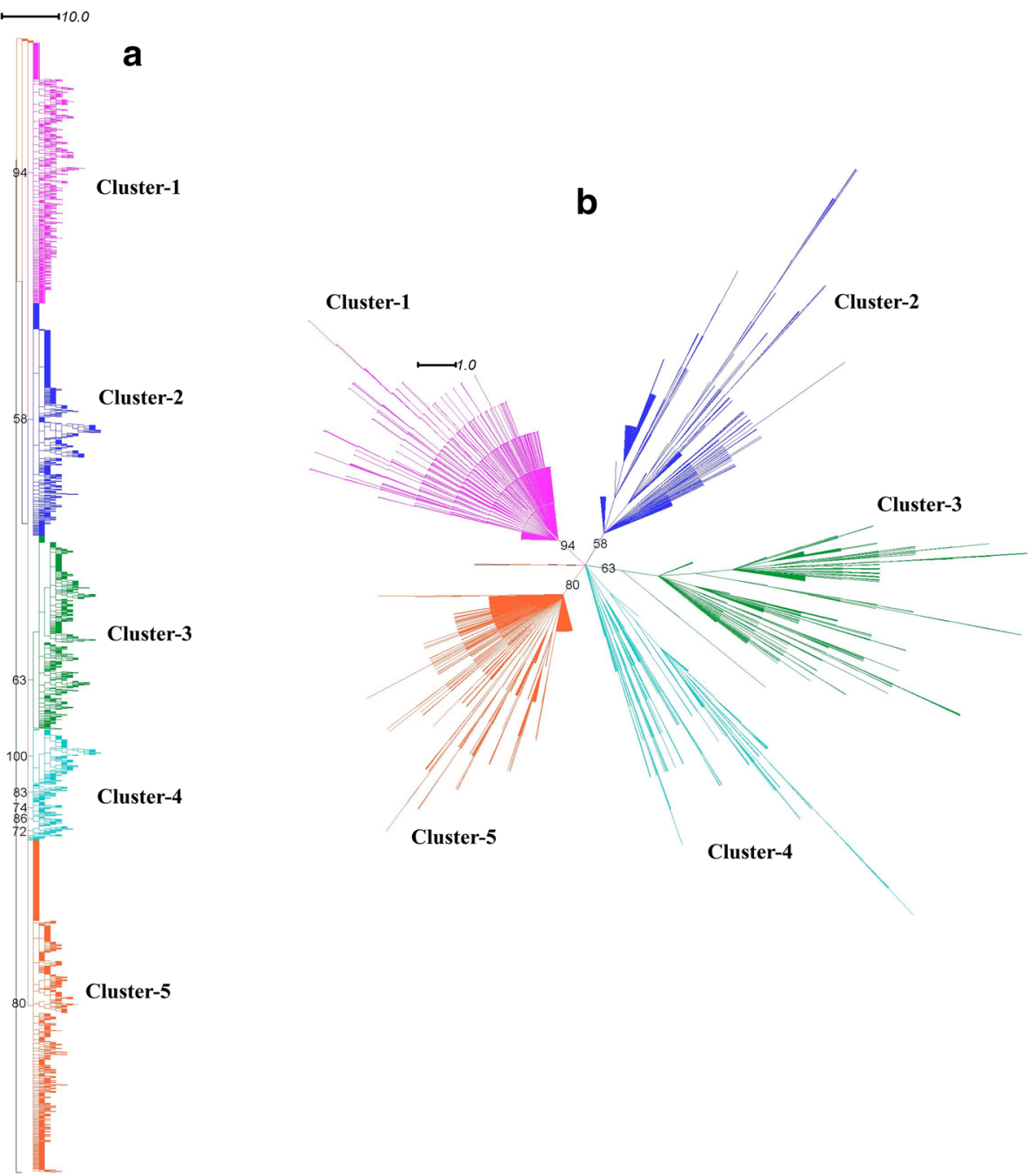

Fig. 1 a Cladogram of LuxS protein sequences of different bacterial species constructed by Maximum Likelihood method with 100 bootstrap replicates; $\mathbf{b}$ Dendrogram of LuXS protein sequences constructed with above mentioned methods

horizontal gene transfer events, such as in Helicobacter pylori, which belongs to Epsilon-Proteobacteria. LuxS sequence of $H$. pylori is grouped in the second cluster. This observation of distinct clustering of $H$. pylori LuxS sequences (in Cluster-2) could be reasoned that H. pylori may have acquired LuxS gene from another species, within Cluster-2, by means of horizontal or lateral gene transfer. Another such case is grouping of many LuxS sequences from Actinobacteria in Cluster-3. These sequences are of various species from Bifidobacteria, Acidaminococcus and Gardenerella genera. This observation is in line with previous study on LuxS proteins in Bifidobacterium genus, even though the study suggests the close homology of Bifidobacterium LuxS sequences with LuxS sequence of Vibrio harveyi [14].

We also analysed the LuxS sequence clusters according to their habitat distribution (Additional files 3 and 5) and found that most of the sequences in Cluster-1 were gut endosymbionts in mammals and other animals, while many sequences in Cluster-2 were halophiles, alkaliphiles and psychrophiles. Many LuxS sequences in Cluster-3 are from plant/food associated bacteria and many LuxS sequences were of extremophilic origin in Cluster-4 (including thermophiles, alkaliphiles, deep-sea hydrothermal vent residents and psychrophiles). In Cluster-5, many LuxS sequences were gut-associated bacteria, marine inhabitants or were plant pathogens. In general, LuxS sequences of pathogenic bacteria were distributed across Clusters-1, 2, 3 and 5, but were not observed in Cluster-4. The grouping of many Actinobacterial and Firmicute sequences in different clusters hint at the evolution of the protein through a series of lateral gene transfers. Nevertheless, the classification of LuxS sequences according to their habitat distribution suggests that LuxS protein may also have evolved through convergent evolution, particularly in case of LuxS sequences of Firmicute species. 


\section{Gene synteny analyses of LuxS genes}

It is well-known that genes that are involved in the pathway of quorum sensing occur as chromosomal neighbours. Hence, we examined for genes neighbouring to LuxS genes in different bacterial genomes. We observed that majority of genes neighbouring to LuxS genes (present upstream, as well as downstream of LuxS gene) are those annotated as hypothetical proteins (Additional files 6 and 7). We performed Multiple Sequence Alignment (MSA) of the hypothetical proteins to examine whether these genes, coding for hypothetical proteins, have a significant common evolutionary origin, and found that they do not have any identical or similar sequences, and thus remain different from each other. However, the hypothetical protein coding genes were functional neighbours, i.e., they were a component of an operon along with the LuxS gene in only 6 of 36 genomes. We also observed that genomes of phyla Gamma-Proteobacteria, and Bacteroidetes tend to have similar syntenies, though this pattern is not observed in all the phyla (Additional file 6). All members of Gamma-Proteobacteria have gshA gene (coding for Glutamine-cysteine ligase) upstream of LuxS, and both members of the phylum Bacteroidetes have rpsO (coding for Ribosomal subunit protein S15) downstream of LuxS gene. One possible reason for this may be that there is an evolutionary constraint for species of Gamma-Proteobacteria and Bacteoidetes to retain the order of genes conserved. The biological aspect behind this constraint has to be further investigated.

Moreover, in many species considered in our analysis, genes that are important for survival of the organism are present downstream of LuxS gene, such as gene coding for DNA-protecting protein in Geobacillus thermodenitrificans (Radiation-resistant bacteria of Bacillales order), another on cell wall-associated hydrolase in Clostridium acetobutylicum and third for hemolysin in Vibrio cholerae (Additional file 6). These genes may be unique to bacterial-species in question, or hold high functional significance for the organism in question.

\section{Motif analyses of LuxS sequences from different clusters}

We next examined unique conserved motifs in LuxS sequences of each cluster obtained from the phylogenetic tree. We found the presence of motifs conserved in all the five clusters, and motifs unique to each cluster were also seen (Fig. 2). For example, structural motif corresponding to the first $\alpha$-helix of LuxS protein is conserved in LuxS proteins of the entire five clusters. However, Cluster-1 has 15 conserved motifs, Cluster-2 has 8 conserved motifs, Cluster- 3 each has 6 conserved motifs, Cluster- 4 has 9 motifs and Cluster- 5 has 6 conserved motifs.
Surface electrostatic potential analysis of LuxS protein of Bacillus subtilis 168 (PDB Id: 1J98)

The electrostatic surface potential was next analyzed using the crystal structure of LuxS protein of Bacillus subtilis (PDB id: 1598). We observed the presence of a patch of positively charged potential, comprising residues on the first and second $\beta$-strand (K25, K35 and R39) (Fig. 3, Additional file 8). It has also been observed in the crystal structure of LuxS protein of Deinococcus radiodurans that these residues are involved in conformational changes that facilitate binding of the substrate to the active site [15]. Therefore, these interactions may contribute to the structural stability of the LuxS homodimer. Negative electrostatic potential can also be seen around the metal-ion binding motif (HXXEH), and on the region behind the metal-ion binding motif, that corresponds to the surface-exposed residues of second helix, and a mixture of negative, as well as positive electrostatic potentials can be observed on surface-exposed residues of third helix. Presence of negative electrostatic potentials on the residues near the $\mathrm{N}$-terminus further confirms the fact that these residues may facilitate binding of the substrate in the dimer form of LuxS protein, as discussed by Ruzheinikov and coworkers [8]. It has been reported that a small RNA molecule MicA is involved in biofilm formation in Salmonella enterica and is located in close proximity to LuxS gene [16]. It has also been reported that certain peptides can bind to LuxS protein and inhibit its activity partially in Streptococcus suis [17]. Our results might suggest the possible mode of interaction of LuxS proteins with these molecules.

\section{Homology modelling of representative LuxS protein sequences from each cluster and surface electrostatic potential analyses}

We next generated homology models of LuxS protein sequences of Borrelia burgdorferi, Amphibacillus jilinensis, Lactobacillus plantarum, Truepera radiovictrix and Vibrio harveyi, belonging to Clusters 1-5, respectively, using MODELLER program [18-20] (Additional files 9 and 10). Twenty models were generated for each sequence, starting from LuxS crystal structure of B. subtilis as a template retrieved from PDB database [21]. The best model was selected based on MODELLER/DOPE scores. Multiple structural alignment of the homology models has shown that motifs having secondary structural elements have similar spatial orientation. In contrary, $\mathrm{N}$ - terminus and $\mathrm{C}$-terminus regions (shown in blue and red respectively in Additional file 10), the region between first $\alpha$-helix and third $\beta$-strand, as well as the loop regions between second and third $\alpha$-helices (shown in green and orange, respectively, in Additional file 10) were found to have structural deviations. Around 


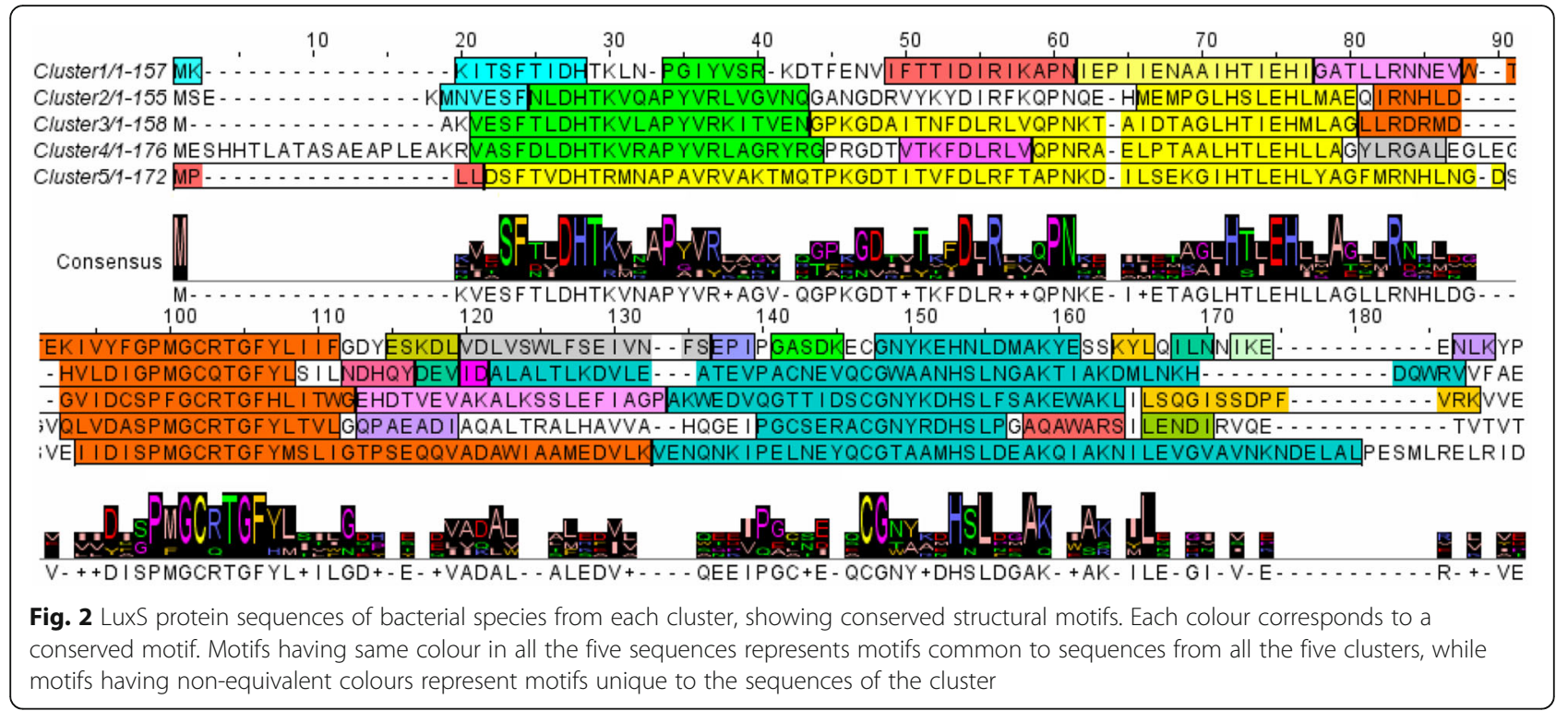

$67 \%$ of residues of reference structure were found to be equivalent, when the superposed homology models were compared at $0.8 \AA$ threshold.

A multiple structural alignment of homology models with LuxS crystal structure of B. subtilis as template, followed by clustering on the basis of Lesk-Hubbard (L$\mathrm{H})$ structural parameters, shows the formation of two main clusters of homology models (Additional file 11). Homology models of LuxS proteins from Vibrio harveyi, Amphibacillus jilinensis and LuxS crystal structure of Bacillus subtilis tend to form one cluster, which implies that these three proteins are likely to be similar to each other. This is also supported by their higher identities in their sequences (average sequence identity of $43 \%$ ). Similarly, homology models of LuxS proteins of Lactobacillus plantarum and Truepera radiovictrix formed a distinct cluster, leaving behind model of LuxS protein of Borrelia burgdorferi unclustered with other protein models. Formation of clusters in LH plot provides some perspective on the structural distances of LuxS protein. The N-terminal region of $T$. radiovictrix was found to be structurally dissimilar with respect to same regions of other homology models. Indeed, the model of $T$. radiovictrix tends to be present on one side of the L-H plot.

We next examined the surface electrostatic potentials of the homology models in order to examine any difference in their profiles amongst the models. We observed the presence of negative surface electrostatic potentials at regions corresponding to metal binding motif (HXXEH) at the first helix, and we also observed negative electrostatic potentials on residues of second and third helices in all five homology models, albeit at low conservation. This further supports our inference that the second helix may be an interacting partner with regulatory proteins or small-RNAs, as negative electrostatic potentials seem to be more conserved than
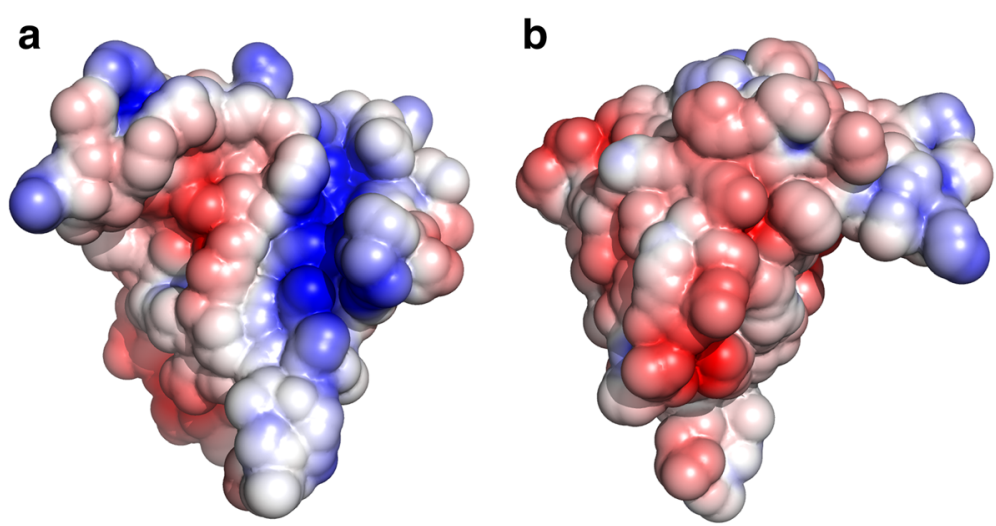

Fig. 3 Structure of LuxS protein of B. subtilis (PDB Id: 1J98) showing surface electrostatic potentials from front view (a) and back view (b) 
positive electrostatic potentials (Additional files 12 and 13). Presence of negative and positive electrostatic potentials on the residues near $\mathrm{N}$-terminus signifies that these residues may be important for the structural stability of the protein, even though this region is structurally dissimilar as seen in the multiple structural alignment. Similarly, presence of negative electrostatic potential on residues next to the metal-ion binding residue (C133 on LuxS protein of B. subtilis) show that these residues are involved in conformational change and interaction with the substrate. Though it was seen in the surface potential map of LuxS protein of $B$. subtilis that some residues in the first and second $\beta$-strand contribute to the structural stability of LuxS homodimer, similar potentials were not observed in the homology models, even though corresponding residues are conserved, as in the block corresponding to R39 of LuxS protein of B. subtilis. These may be electrostatic potentials unique to proteins of each cluster.

\section{Conclusion}

In this study, we investigated the evolution of LuxS protein through a phylogenetic and structural perspective. Molecular phylogeny studies on 3106 LuxS protein sequences, an important enzyme in Autoinducer-2 biosynthesis of different bacteria species, has shown grouping of sequences into five distinct clusters. These LuxS protein sequences appear to be grouped on the basis of their habitats and lifestyles, which may be a case of convergent evolution. Synteny analysis of LuxS genes has shown the presence of large number of neighbouring genes annotated as hypothetical proteins suggesting a broader repertoire of biological functions are yet to be discovered. Furthermore, many genes that may be critical for survival of the organism are present downstream of LuxS gene. On the structural front, surface electrostatic analysis of LuxS protein of B. subtilis shows the presence of regions having positive and negative electrostatic potentials, which contribute to the structural stability of LuxS homodimer and may be the sites of protein-protein and protein-RNA interactions. Homology modelling of LuxS protein sequences from each cluster shows the similarities as well as differences among LuxS proteins of different clusters, which are more clear when the models are subjected to structural alignment and analyses of surface electrostatic potentials. Structural alignment showed the grouping of models into two clusters, which may provide some clues about evolution of LuxS protein from a structural perspective. Molecular phylogeny analysis of LuxS protein on evolutionary as well as structural perspective has yielded some insights into the evolution of enzymes involved in biosynthesis of
Autoinducers. However, further studies are required to obtain a clearer picture of evolution of quorumsensing apparatus in bacteria.

\section{Methods \\ Sequence search}

The LuxS protein sequence of Bacillus subtilis 168 (UniProt Id: O34667) was used as a query to retrieve LuxS sequences of different bacterial phyla (Bacteroidetes, Chloroflexi, Dienococcus-Thermus, Fusobacteriales, Alpha-Proteobacteria and Haloplasmatales) using BLASTp program of NCBI [22]. The resulting 20 sequences from the BLASTp sequence searches were used as queries again to search for other LuxS homologues against Non-Redundant database using phmmer program (version 1.4) [13]. The results were retrieved and merged to eliminate redundancy.

\section{Phylogeny of LuxS protein}

The non-redundant LuxS homologues were then aligned by PROMALS3D multiple alignment web server [23]. The consensus regions were identified, and nonconsensus regions were removed using Jalview (version: 2.8.2) [24]. The phylogenetic tree was constructed by Maximum Likelihood method using RAxML program [25] with 100 bootstrap replicates (version 8.0.0). The resulting tree was visualised and edited using Dendroscope program (version 3.2.10) [26].

\section{Gene synteny analysis of LuxS gene}

The gene locations of different bacterial species were examined in BioCyc database collection (version 19.0) [27] in order to examine the location of LuxS genes in different bacterial genomes. Information regarding gene position, location in operon, particulars of genes located upstream and downstream of LuxS gene was noted, SCOP classification was obtained by SUPERFAMILY HMM library and genome assignment server (version 1.75) $[28,29]$. The hypothetical proteins were aligned using ClustalW algorithm [30] of MEGA6 program [31] to analyse the similarities between the hypothetical proteins present downstream of LuxS gene,

\section{Motif analysis of LuxS protein}

The unique gi-identification numbers of sequences in each cluster were used as search terms to retrieve the corresponding sequences in fasta format from the NCBI-protein database. The retrieved sequences were analysed for conserved structural motifs using MOTIFS program [32]. The motifs were mapped to MSA of the protein sequences from each cluster generated by $\mathrm{T}$ coffee program [33] using Jalview program (version 2.8.2) [24]. 


\section{Analysis of surface electrostatic potentials of Bacillus subtilis LuxS protein (PDB Id: 1J98)}

Crystal structure of LuxS protein of B. subtilis was submitted to the PDB2PQR web server $[34,35]$ and surface electrostatic potential calculations were performed using Adaptive Poisson Boltzmann Solver (APBS) plugin [36] of Pymol program [37]. The results were visualised using the Pymol program [37], and corresponding residues having positive and negative electrostatic potentials were mapped using Pymol program [37].

\section{Homology modelling of LuxS proteins of different species and analyses of surface electrostatic potential analyses} Query LuxS protein sequences (marked forest green in the LuxS phylogenetic tree showing taxonomic distribution) were selected from each cluster of the phylogenetic tree. The sequences were retrieved in PIR format. The sequences were searched for closest homologues in PDB database [21] using NCBI-BLASTp search program [22], and resulting highest scoring hit was used as template for modelling. 20 homology models were obtained from MODELLER program (version 9.14) [18-20]. The models were evaluated and the best model was chosen based on MODELLER scores. Ramachandran plot of the candidate model was mapped by Rampage program [38] (Additional file 10). Furthermore, the resulting homology models were aligned using MUSTANG multiple structural alignment server [39] with crystal structure of LuxS protein of B. subtilis (PDB Id: 1J98) used as reference. The superposed structure was visualised using Pymol program [37], and the Lesk-Hubbard plot of $C^{\alpha}$ atoms vs. RMSD and sieved structure of reference protein (Crystal structure of LuxS protein of B. subtilis) set to $0.8 \AA$ threshold was retrieved (Additional file 12).

$\mathrm{PQR}$ results were obtained for the best homology model of cluster representatives, exactly as done for the crystal structure. The multiple structure-based sequence alignment of homology models, generated by MUSTANG structural alignment server [39], was used to map these potentials to their respective residues using Jalview program (version 2.8.2) [24].

\section{Additional files}

Additional file 1: Table containing Number of hits obtained from each sequence searches and phylum-wise distribution of LuxS sequences obtained from phmmer searches. (XLSX $39 \mathrm{~kb}$ )

Additional file 2: Table showing Taxonomic distribution of LuxS sequences in bacteria. (XLSX $38 \mathrm{~kb}$ )

Additional file 3: Table showing Gene-synteny information of LuxS genes of different species. (XLSX $41 \mathrm{~kb}$ )

Additional file 4: Phylogenetic tree of LuxS sequences showing taxonomic distribution of sequences. Colour codes: Blue: Firmicutes; Pink: Actinobacteria; Yellow: Bacteroidetes; Cyan: Spirochaetes; Red: Proteobacteria;
Brown: Fusobacteria; Grass Green: Deinococcus-Thermales; Forest green: Query sequences selected for homology modelling. (PDF $2242 \mathrm{~kb}$ )

Additional file 5: Phylogenetic tree of LuxS sequences showing habitat distribution of sequences. Colour codes: Pink: Gut/Faecal microorganisms; Purple: Animal and Human pathogens; Brown: Soil-dwelling bacteria; Yellow-green: Food-associated bacteria; Forest-green: Plant-associated bacteria; Sky blue: Halophiles; Navy blue: Alkaliphiles; Cyan: Psychrophiles; Orange: Thermophiles; Grey: Radiation-resistant bacteria; Yellow: Hydrothermal vent inhabiting bacteria; Lignt blue: Marine bacteria; Blue-green: Plant pathogens; Black: Habitat information not available (PDF 108 kb)

Additional file 6: Cladograms of LuxS protein sequences showing (A) taxonomic distribution of species (Colour code: Blue: Firmicutes; Pink: Actinobacteria; Yellow: Bacteroidetes; Cyan: Spirochaetes; Red: Proteobacteria; Brown: Fusobacteria; Green: Deinococcus-Thermales) and habitat distribution of bacteria possessing the LuxS proteins (B), colour codes: Pink: Gut/Faecal microorganisms; Purple: Animal and Human pathogens; Brown: Soil-dwelling bacteria; Yellow-green: Food-associated bacteria; Forest-green: Plant-associated bacteria; Sky blue: Halophiles; Navy blue: Alkaliphiles; Cyan: Psychrophiles; Orange: Thermophiles; Grey: Radiation-resistant bacteria; Yellow: Hydrothermal vent inhabiting bacteria; Light blue: Marine bacteria; Blue-green: Plant pathogens; Black: Habitat information not available). (PDF $2242 \mathrm{~kb}$ )

Additional file 7: Pie chart showing distribution of SCOP protein superfamily-coding genes present as a component of an operon (A), present upstream (B) and present downstream (C) with respect to the LuxS gene in different bacterial species. (PDF $553 \mathrm{~kb}$ )

Additional file 8: Pymol session file showing APBS map of LuxS protein of Bacillus subtilis (PDB Id: 1J98). As per the usual convention, blue regions correspond to the residues having positive surface electrostatic potentials, while red regions correspond to the residues having negative surface electrostatic potentials. (PSE $29862 \mathrm{~kb}$ )

Additional file 9: Folder containing homology models of LuxS proteins of Borrelia burgdorferi (Cluster-1), Amphibacillus jilinensis (Cluster-2), Lactobacillus plantarum (Cluster-3), Truepera radiovictrix (Cluster-4) and Vibrio harveyi (Cluster5); Multiple structural alignment of homology models; Sieved structure of LuxS protein of $B$. subtilis against $0.8 \AA$ threshold from the multiple structural alignment. (ZIP $677 \mathrm{~kb}$ )

Additional file 10: Homology models of LuxS of representatives from the clusters and Ramachandran plots of homology models. (ZIP $936 \mathrm{~kb}$ )

Additional file 11: Lesk-Hubbard plot of the multiple structural alignment of the homology models. (PDF $40 \mathrm{~kb}$ )

Additional file 12: Multiple structure-based sequence alignment of homology models of LuxS protein sequences, showing surface electrostatic potentials. Residues labelled in red are regions with negative surface potentials; residues labelled in blue are regions having positive surface electrostatic potentials. (PDF 157 kb)

Additional file 13: Folder containing pymol session files showing APBS maps of the homology models. Colour coding is same as in Additional file 8. (ZIP $35165 \mathrm{~kb}$ )

\section{Abbreviations}

Al: Autoinducer; DOPE: Discrete Optimized Protein Energy; HMM: Hidden Markov Model; MSA: Multiple Sequence Alignment; NCBI: National Center for Biotechnology Information; PROMALS3D: PROfile Multiple Alignment with predicted Local Structures and 3D constraints; RAxML: Randomized Axelerated Maximum Likelihood

Funding

The authors acknowledge financial and infrastructural support from NCBS-TIFR.

Availability of data and materials

The phylogeny data, provided in Fig. 1, can also be accessed from Treebase at http://purl.org/phylo/treebase/phylows/study/TB2:S19637.

Authors' contributions

RS conceived and designed the study. SNP and RMR performed the study. RS, SNP and RMR analysed the data. RMR wrote the first draft of the 
manuscript. RS revised the final version of the text. All three authors read and approved the final manuscript.

\section{Competing interests}

The authors declare that they have no competing interests.

\section{Consent for publication}

Not applicable.

\section{Ethics approval and consent to participate}

Not applicable.

\section{Author details}

${ }^{1}$ National Centre for Biological Sciences, Tata Institute of Fundamental Research, GKVK campus, Bellary Road, Bangalore 560065, India. ${ }^{2}$ Division of Biological Sciences, School of Natural Sciences, Bangalore University, Bangalore 560056, India.

\section{Received: 12 December 2015 Accepted: 9 August 2016} Published online: 20 September 2016

\section{References}

1. Nealson KH. Autoinduction of bacterial luciferase. Occurrence, mechanism and significance. Arch Microbiol. 1977;112(1):73-9.

2. de Kievit TR, Iglewski BH. Bacterial Quorum Sensing in pathogenic relationships. Infect Immun. 2000;68(9):4839-49.

3. Chen X, Schauder S, Potier N, Van Dorsselaer A, Pelczer I, Bassler BL, Hughson FM. Structural identification of a bacterial quorum-sensing signal containing boron. Nature. 2002;415(6871):545-9.

4. Parveen N, Cornell KA. Methylthioadenosine/S-adenosylhomocysteine nucleosidase, a critical enzyme for bacterial metabolism. Mol Microbiol. 2011;79(1):7-20.

5. Schauder S, Shokat K, Surette MG, Bassler BL (2001). The LuxS family of bacterial autoinducers: biosynthesis of a novel quorum-sensing signal molecule. Mol. Microbiol. 41(2): 463-76.

6. Pei D, Zhu J. Mechanism of action of S-ribosylhomocysteinase (LuxS). Curr Opin Chem Biol. 2004:8(5):492-7.

7. Surette MG, Miller MB, Bassler BL. Quorum sensing in Escherichia coli, Salmonella typhimurium, and Vibrio harveyi: a new family of genes responsible for autoinducer production. Proc Natl Acad Sci U S A. 1999; 96(4):1639-44.

8. Ruzheinikov SN, Das SK, Sedelnikova SE, Hartley A, Foster SJ, Horsburgh MJ, Cox AG, McCleod CW, Mekhalfia A, Blackburn GM, Rice DW, Baker PJ. The 1. 2 A structure of a novel quorum-sensing protein, Bacillus subtilis LuxS. J Mol Biol. 2001;313(1):111-22.

9. Hilgers MT, Ludwig ML. Crystal structure of quorum-sensing protein LuxS reveals a catalytic metal site. Proc Natl Acad Sci U S A. 2001; 98(20):11169-74.

10. Lerat $E$, Moran NA. The evolutionary history of quorum-sensing systems in bacteria. Mol Biol Evol. 2004;21(5):903-13.

11. Sun J, Daniel R, Wagner-Döbler R, Zeng AP. Is autoinducer-2 a universal signal for interspecies communication: a comparative genomic and phylogenetic analysis of synthesis and transduction pathways. BMC Evol Biol. 2004:4:36.

12. Duffy $B$, Rezzonico F. Lack of genomic evidence of Al-2 receptors suggests a non-quorum-sensing role for luxS in most bacteria. BMC Microbiol. 2008;8:154.

13. Finn RD, Clements J, Eddy SR. HMMER web server: interactive sequence similarity searching. Nucleic Acids Res. 2011;39(Web Server issue):W31-7.

14. Sun Z, He X, Brancaccio VF, Yuan J, Riedel CU. Bifidobacteria exhibit LuxS dependent autoinducer 2 activity and biofilm formation. PLoS One. 2014; 9(2):e88260.

15. Lewis HA, Furlong EB, Laubert B, Eroshkina GA, Batiyenko Y, Adams JM, Bergseid MG, Marsh CD, Peat TS, Sanderson WE, Sauder JM, Buchanan SG. A structural genomics approach to the study of quorum sensing: crystal structures of three LuxS orthologs. Structure. 2001;9(6):527-37.

16. Kint G, De Coster D, Marchal K, Vanderleyden J, De Keersmaecker SCJ. The small regulatory MicA is involved in Salmonella enterica serovar Typhimurium biofilm formation. BMC Microbiol. 2010;10:276.

17. Han X, Lu C. Biological activity and identification of a peptide inhibitor of LuxS from Streptococcus suis serotype 2. FEMS Microbiol Lett. 2009;294(1): $16-23$.
18. Eswar N, Webb B., Marti-Renom MA, Madhusudhan MS, Eramian D, Shen MY Pieper U, Sali A. Comparative protein structure modeling using MODELLER. Curr Protoc Bioinformatics. Supplement 15;2006: 5.6.1-5.6.31. John Wiley \& Sons, Inc.

19. Sali A, Blundell TL. Comparative protein modelling by satisfaction of spatial restraints. J Mol Biol. 1993;234(3):779-815.

20. Fiser A, Do RK, Sali A. Modelling of loops in protein structures. Protein Sci. 2000;9(9):1753-73.

21. Berman HM, Westbrook J, Feng Z, Gililand G, Bhat TN, Weissing H, Shindyakov IN, Bourne PE. The protein data bank. Nucl Acids Res. 2000;31(1):235-42.

22. Altschul SF, Gish W, Miller W, Myers EW, Lipman DJ. Basic local alignment search tool. J Mol Biol. 1990;215(3):403-10.

23. Pei J, Grishin NV. PROMALS3D: multiple protein sequence alignment enhanced with evolutionary and three-dimensional structural information. Methods Mol Biol. 2014;1079:263-71.

24. Waterhouse AM, Procter JB, Martin DM, Clamp M, Barton GJ. Jalview Version 2-a multiple sequence alignment editor and analysis workbench. Bioinformatics. 2009;25:1189-91. doi:10.1093/bioinformatics/btp033.

25. Stamatakis A. RAxML version 8: a tool for phylogenetic analysis and post analysis of large phylogenies. Bioinformatics. 2014;31(9):1312-3.

26. Huson DH, Scornavacca C. Dendroscope 3: an interactive tool for rooted phylogenetic trees and networks. Syst Biol. 2012;61(6):1061-7.

27. Caspi R, Altman T, Billington R, Dreher K, Foerster H, Fulcher CA, Holland TA, Keseler IM, Kothari A, Kubo A, Krummenacker M, Latendresse M, Mueller LA, Ong Q, Paley S, Subhraveti P, Weaver DS, Weerasinghe D, Zhang P, Karp PD. The MetaCyc database of metabolic pathways and enzymes and the BioCyc collection of Pathway/Genome Databases. Nucleic Acids Res. 2014; 42(Database issue):D459-71.

28. Murzin AG, Brenner SE, Hubbard T, Chothia C. SCOP a structural classification of proteins database for the investigation of sequences and structures. J Mol Biol. 1995:247:536-40.

29. Wilson D, Pethica R, Zhou Y, Talbot C, Vogel C, Madera M, Chothia C, Gough J. SUPERFAMILY-sophisticated comparative genomics, data mining, visualization and phylogeny. Nucleic Acids Res. 2009;37(Database issue): D380-6.

30. Thompson JD, Higgins DG, Gibson TJ. CLUSTAL W: improving the sensitivity of progressive multiple sequence alignment through sequence weighting, position-specific gap penalties and weight matrix choice. Nucleic Acids Res. 1994:22(22):4673-80.

31. Tamura K, Stecher G, Peterson D, Filipski A, Kumar S. MEGA6: molecular evolutionary genetics analysis version 6.0. Mol Biol Evol. 2013;31(12): 2725-31.

32. Chakrabarti S, Bhardwaj N, Anand PA, Sowdhamini R. Improvement of alignment accuracy utilizing sequentially conserved motifs. BMC Bioinformatics. 2004;5:167kl.

33. Notredame, C., D. G. Higgins and J. Heringa (2000). T-Coffee: A novel method for fast and accurate multiple sequence alignment. J Mol Biol 312(1): 205-217.

34. Dolinsky TJ, Czodrowski P, Li H, Nielsen JE, Jensen JH, Klebe G, Baker NA. PDB2PQR: expanding and upgrading automated preparation of biomolecular structures for molecular simulations. Nucleic Acids Res. 2007;35(Web Server issue):W522-5.

35. Dolinsky TJ, Nielsen JE, McCammon JA, Baker NA. PDB2PQR: an automated pipeline for the setup, execution, and analysis of Poisson-Boltzmann electrostatics calculations. Nucleic Acids Res. 2004:32:W665-7.

36. Baker, N. A., D. Sept, S. Joseph, M. J. Holst and J. A. McCammon (2001). Electrostatics of nanosystems: application to microtubules and the ribosome. Proc Natl Acad Sci U S A 98(18): 10037-10041.

37. DeLano WL. The PyMOL Molecular Graphics System. San Carlos, CA, USA: DeLano Scientific; 2002.

38. Lovell SC, Davis IW, Arendall III WB, de Bakker PIW, Word JM, Prisant MG Richardson JS, Richardson DC. Structure validation by C-alpha geometry: phi, psi and C-beta deviation. Proteins Struct Funct Genet. 2002;50:437-50.

39. Konagurthu AS, Reboul CF, Schmidberger JW, Irving JA, Lesk AM, Stuckey PJ, Whisstock JC, Buckle AM. MUSTANG-MR structural sieving server: applications in protein structural analysis and crystallography. PLoS One. 2010;5(4):e10048. 